\title{
Diseño, implementación y análisis económico de un sistema fotovoltaico conectado a la red (SFCR) de 3 kW con conversores cc/cc analizado en las condiciones geográficas y climatológicas la ciudad de J uliaca
}

Design, implementation and economic analys is of a $3 \mathrm{~kW}$ photovoltaic system connected to the
grid (SFCR) with dc/dc converters analyzed under geographical and climatological conditions the
city of J uliaca
city of J uliaca

\author{
Vanesa Chávez Chambi \\ v.chavez.ch@ unaj.edu.pe - Universidad Nacional de J uliaca \\ Reynaldo Condori Yucra \\ rcondori@ unaj.edu.pe - Universidad Nacional de J uliaca \\ Pedro Yulian Puma Roque \\ py.puma@ unaj.edu.pe - Universidad Nacional de J uliaca \\ Norman Jesús Beltrán Castañón \\ nbeltran@unaj.edu.pe - Universidad Nacional de J uliaca
}

Recibido el 18/08/20 | Aceptado el 01/10/20

DOI: https://doi.org/10.47190/nric.v3i1.122

\section{Resumen}

La siguiente investigación se desarrolló en el marco del proyecto de investigación aplicada y desarrollo tecnológico ganador de los fondos concursables financiado por el FONDECYT-Banco Mundial, con Contrato N ${ }^{\circ} 180-2018$ FONDECYT-BM-IADT-AV. En donde se realizó el Diseño, Implementación y el Análisis Económico del SFCR con conversores cc/cc, para una potencia instalada de $2.96 \mathrm{~kW}$. Implementado en el pabellón del Instituto de Energías Renovables y Eficiencia Energética de la Universidad Nacional de Juliaca (UNAJ) sede Ayabacas. Las horas solar pico (HSP) son de $6.0 \mathrm{kWh} / \mathrm{m}^{2} /$ día promedio anual, la temperatura ambiental de $18^{\circ} \mathrm{C}$ promedio anual. La producción de energía es de $5,650.99 \mathrm{kWh} / \mathrm{año}$, la tarifa regulada es la misma de la concesionaria S/. 0.6545kWh/mes, Los indicadores económicos del SFCR con inversión inicial de S/.29,417.55 para un tiempo de operación de 25 años, dan como resultado, el VPN de S/. 12,806.0, ratio de 1.60; TIR de $13 \%$, y el periodo de recuperación de la inversión de 12 años; dando lugar a que el proyecto sea viable económicamente.

Palabras claves: Sistema fotovoltaico, conversores cc/cc, conexión a red.

\begin{abstract}
The following research was developed within the framework of the applied research and technological development project that was awarded the competitive funds, financed by FONDECYT-World Bank, with Contract No. 180-2018FONDECYT-BM-IADT-AV. Where the Design, Implementation and Economic Analysis of the SFCR with cc/cc converters was carried out, for an installed power of $2.96 \mathrm{~kW}$. Implemented in the pavilion of the Institute of Renewable Energies and Energy Efficiency of the National University of Juliaca (UNAJ), Ayabacas headquarters. Peak solar hours (HSP) are $6.0 \mathrm{kWh} / \mathrm{m} 2$ / day average per year, the ambient temperature is $18^{\circ} \mathrm{C}$ average per year. Energy production is $5,650.99 \mathrm{kWh} /$ year, the regulated rate is the same as that of the concessionaire $\mathrm{S} / .0 .6545 \mathrm{kWh} / \mathrm{month}$. The economic indicators of the SFCR with an initial investment of S /.29, 417.55 for an operating time of 25 years, result in the NPV of S /. 12.806.0, ratio 1.60; TIR of 13\%, and the investment recovery period of 12 years; resulting in the project being economically viable.
\end{abstract}

Keywords: Photovoltaic system, DC/DC converters, network connected.

Como citar: Chávez-Chambi, V., Condori-Yucra, R., Puma-Roque, P.Y. \& Beltrán-Castañón, N.J. (2020). Diseño, implementación y análisis económico de un sistema fotovoltaico conectado a la red (SFCR) de $3 \mathrm{~kW}$ con conversores cc/cc analizado en las condiciones geográficas y climatológicas la ciudad de Juliaca. ÑAWPARISUN - Revista de Investigación Científica, 3(1), 13-21. 


\section{Introducción}

Un SFCR es un sistema cuya función es producir energía eléctrica en condiciones adecuadas para poder ser inyectada en la red convencional, se compone del generador fotovoltaico, un inversor $\mathrm{DC} / \mathrm{AC}$ y un conjunto de protecciones eléctricas. La energía producida por este sistema será consumida parcial o totalmente en las cercanías, y la energía sobrante será inyectada en la red para su distribución a otros puntos de consumo. En este caso, el diseño no necesita considerar un consumo a satisfacer. Con este mecanismo, el objetivo del diseñador es que la producción anual del sistema sea la máxima posible sin tomar en consideración los consumos cercanos (Perpiñán et al., 2012).

En la disertación de Lopes (2006), se detalla los tipos y técnicas de conmutación de los convertidores estáticos cc/cc y cc/ca. La energía procedente de los paneles fotovoltaicos es procesada en los convertidores cc/cc boost, estos convertidores se controlan para rastrear el punto de máxima potencia y también para eliminar selectivamente los armónicos, finalmente con todas las simulaciones y resultados experimentales demostraron que las topologías y técnicas adoptadas para el control de los convertidores $\mathrm{cc} / \mathrm{cc}$ y $\mathrm{cc} / \mathrm{ca}$ son perfectamente empleadas en el control del sistema experimental.

En la disertación de Teles (2017), se evalúo experimentalmente 3 tecnologías, las cuales son: inversor string, microinversor y conversor cc/cc, para la aplicación de las diferentes topologías de interconexión de generadores fotovoltaicos a la red, para esto se utilizaron diferentes sistemas instalados en el laboratorio de GEDAE / UFPA, región norte de Brasil, los resultados obtenidos muestran que el convertidor cc/cc presentó desempeños superiores a los demás sistemas monitoreados, en períodos de operación sin anomalías.

En el país no se tienen investigaciones de sistemas fotovoltaicos conectados a red con conversores $\mathrm{cc} / \mathrm{cc}$ que muestren resultados del uso de este tipo de conversor, por lo cual se propone evaluar al conversor $\mathrm{cc} / \mathrm{cc}$ teniendo las condiciones climáticas de irradiación solar y áreas geográficas ideales, a esto se suma que las nuevas tecnologías son cada vez más eficientes y económicas, además de obtener energía limpia, segura y sustentable.

\section{Teoría general de los conversores cc/cc}

Los conversores cc/cc sirven para transformar una tensión de entrada continua, generalmente de valor fijo, en una tensión de salida también de corriente continua.(Domingo, 2017).

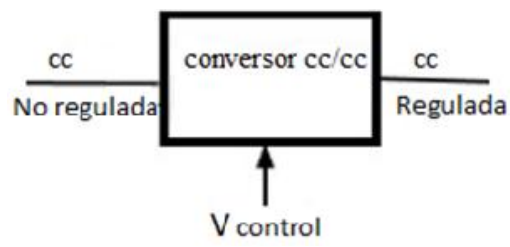

Figura 1. Esquema general de los conversores CC/CC (Domingo, 2017).
El conversor buck - boost es un tipo de conversor cc/cc que puede presentar una tensión mayor o menor en la salida con respecto a la tensión de entrada, la tensión de salida se controla modificando el ciclo de servicio del transistor (Domingo, 2017).

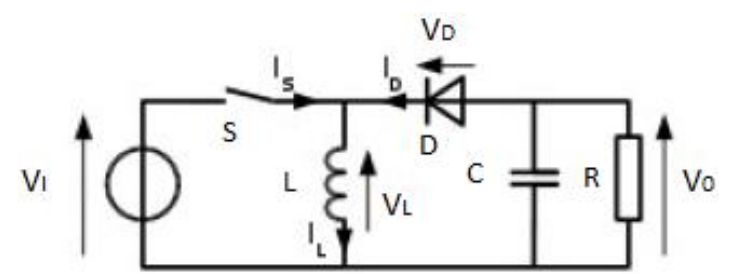

Figura 2. Esquema de un conversor Buck-Boost (Domingo, 2017).

\section{Arquitectura de conexión con conversores cc/cc}

La Figura muestra la potencia de salida de dos módulos fotovoltaicos colocados en serie dependiendo de la corriente. La curva roja muestra la potencia proporcionada por la asociación de estos módulos en serie si no se utilizan medidas adicionales aparte del uso del diodo. Tenga en cuenta que un módulo tiene una corriente de cortocircuito de $10 \mathrm{~A}$ y el otro tiene una corriente de cortocircuito de $8 \mathrm{~A}$. La corriente de potencia máxima está limitada por el módulo FV que produce menos corriente, evitando que el módulo tenga más potencia disponible para operar en su punto de máxima potencia.

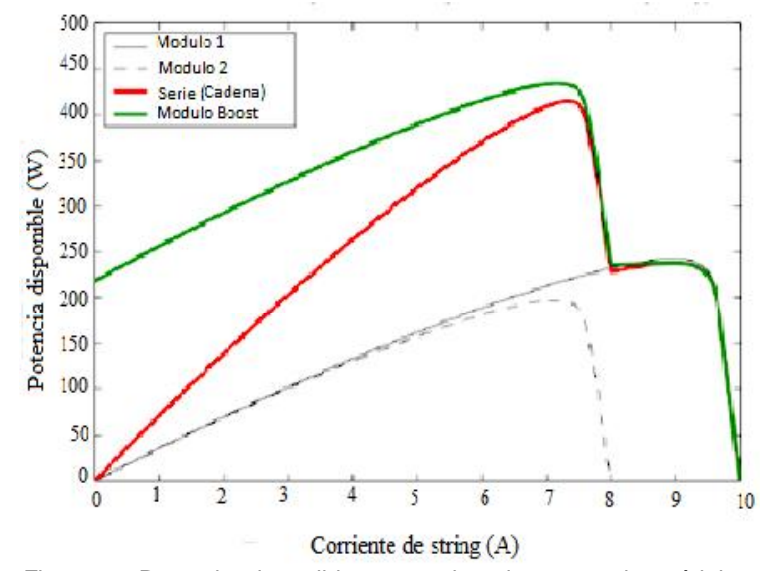

Figura 3. Potencia de salida proporcionada por cada módulo y generador fotovoltaico adaptado de Johns, Le, \& Seeman (2008).

En la Figura, si se agrega un conversor Boost al panel con la mayor potencia disponible para reducir su corriente mientras aumenta su voltaje, ambos módulos fotovoltaicos pueden operar en su punto de máxima potencia. La curva verde muestra la energía producida por los dos módulos conectados en serie cuando el más potente tiene instalado un conversor Boost. En este escenario particular, se puede lograr una mejora de $20 \mathrm{~W}$ (Johns et al., 2008).

En el último caso, se utiliza un "inversor central" para transferir la potencia disponible en la salida de asociación del conversor cc/cc hasta el punto de conexión a la red eléctrica, donde la Figura 4 ilustra bien esta configuración. 


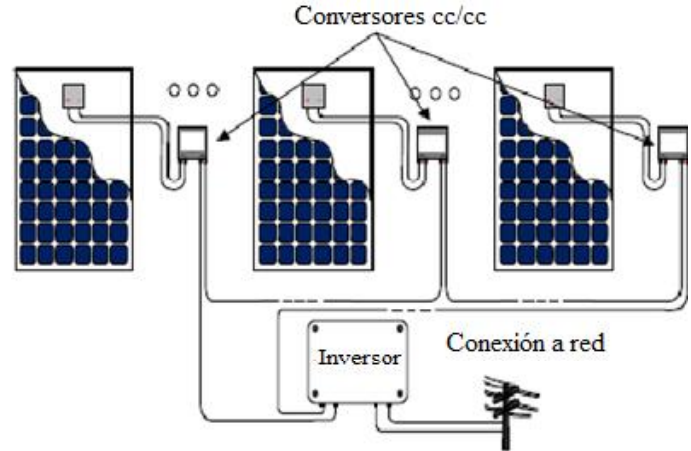

Figura 4. Diagrama esquemático de configuración con conversores cc/cc adaptado de Teles (2017).

Una de las principales ventajas de esta arquitectura es la reducción significativa de las pérdidas por dispersión de parámetros eléctricos, ya que cada módulo FV opera en su punto individual de máxima potencia, independientemente de los otros módulos del sistema (Teles, 2017).

\section{Materiales y métodos}

\section{Módulos fotovoltaicos}

El Sistema fotovoltaico contará 8 módulos fotovoltaicos monocristalinos de $370 \mathrm{Wp}$ en la marca BAUER con una eficiencia nominal de $19 \%$.

Tabla 1.

Parámetros eléctricos y térmicos del módulo fotovoltaico, adaptado de la ficha tecnica del fabricante (BAUER, 2019).

\begin{tabular}{cc}
\hline \multicolumn{2}{c}{ Parámetros eléctricos (STC) } \\
\hline P mp & $370 \mathrm{~W}$ \\
Imp & $9.23 \mathrm{~A}$ \\
Vmp & $40.1 \mathrm{~V}$ \\
ISC & $9.95 \mathrm{~A}$ \\
VoC & $48.3 \mathrm{~V}$ \\
\hline Parámetros térmicos & $45{ }^{\circ} \mathrm{C}$ \\
\hline TNOC & $+0.02973 \% /{ }^{\circ} \mathrm{C}$ \\
$\alpha$ & $-0.38038 \% /{ }^{\circ} \mathrm{C}$ \\
\hline
\end{tabular}

\section{Conversor cc/cc}

El Sistema fotovoltaico estará compuesto también por 8 Conversores $\mathrm{cc} / \mathrm{cc}$ en la marca Solaredge modelo P370, eficiencia ponderada a $98,8 \%$,

Tabla 2.

Parámetros eléctricos del conversor cc/cc P370 adaptado del ficha tecnica del fabricante (Solar Edge, 2019).

\begin{tabular}{|c|c|}
\hline \multicolumn{2}{|c|}{ Parámetros eléctric os (entrada) } \\
\hline$P_{\text {nominal }}$ & $370 \mathrm{~W}$ \\
\hline lentrada max & 13.75 Acd \\
\hline Ventrada máx. & $60 \mathrm{Vcd}$ \\
\hline Isc & 11 Acd \\
\hline Rango operativo MPPT & $8-60 \mathrm{Vdc}$ \\
\hline \multicolumn{2}{|c|}{ Salida durante el funcionamiento (conectado al inversor en operación) } \\
\hline Isalida max. & 15 Acd \\
\hline$V_{\text {salida max. }}$ & $60 \mathrm{Vcd}$ \\
\hline \multicolumn{2}{|c|}{ Salida durante modo standBy (Desconectado al inversor) } \\
\hline$V_{\text {salida de seguridad cc-cc }}$ & $1 \mathrm{Vcd}$ \\
\hline
\end{tabular}

\section{Inversor cc /ca}

Para este sistema usaremos un inversor monofásico también fabricado por Solaredge modelo SE3000H, estos inversores están específicamente diseñado para trabajar con optimizadores de energía o también conocidos como conversores cc/cc. En la Tabla 3 se muestran los datos de la placa del inversor SE3000H.

Tabla 3.

Características eléctricas del inversor SE $3000 \mathrm{H}$, adaptado de la ficha técnica del fabricante (Solaredge, 2019). $\eta_{\text {inversor }}=99.2 \%$, y consumo de $2.5 \mathrm{~W}$

\begin{tabular}{lcc}
\hline \multicolumn{3}{c}{ Parámetros eléctricos de entrada CC } \\
\hline Pmáx & $4650 \mathrm{~W}$ \\
Imáx & $9 \mathrm{Acd}$ \\
Vmáx & $480 \mathrm{Vcd}$ \\
Vnom & $380 \mathrm{Vcd}$ \\
\hline \multicolumn{3}{c}{ Parámetros eléctricos de salida CA } \\
\hline Pnom & $3000 \mathrm{VA}$ \\
Pmax & $3000 \mathrm{VA}$ \\
Vnom & $220 / 230 \mathrm{Vca}$ \\
Frecuencia & $50 / 60 \mathrm{~Hz}$ \\
Imáx & $14 \mathrm{~A}$ \\
\hline
\end{tabular}

\section{Metodología}

La metodología abordada en esta investigación es del tipo analítico descriptiva, porque se establece una descripción completa; dado que se generarán datos de las características técnicas y económicas del sistema solar fotovoltaico, además se relacionaron todos los datos obtenidos del diseño. Para realizar el diseño se tomarán datos referentes de la NASA, SENAMHI y cálculos empíricos de proyectos anteriores. Se realiza la implementación del sistema fotovoltaico de acuerdo al diseño realizado anteriormente. Seguidamente se realiza el análisis económico del sistema fotovoltaico, con indicadores de VAN, TIR, Periodo de recuperación y beneficio/costo.

\section{Arquitectura de conexión y diseño eléctrico}

Se realizó el diseño de la arquitectura de instalación eléctrica del sistema fotovoltaico con conversor cc/cc, diseñando la parte de protección tanto en el lado CC y CA.

\section{Normas aplicables}

- IEC 61215: "Terrestrial photovoltaic (PV) modules with crystalline solar cells -Design qualification and type approval"

- IEC 61724-1: "Photovoltaic system performance Part 1: Monitoring"

- Norma DGE: Especificación técnica del sistema fotovoltaico y sus componentes electrificación rural"

- Código Nacional de Electricidad: "Utilización 2006" 


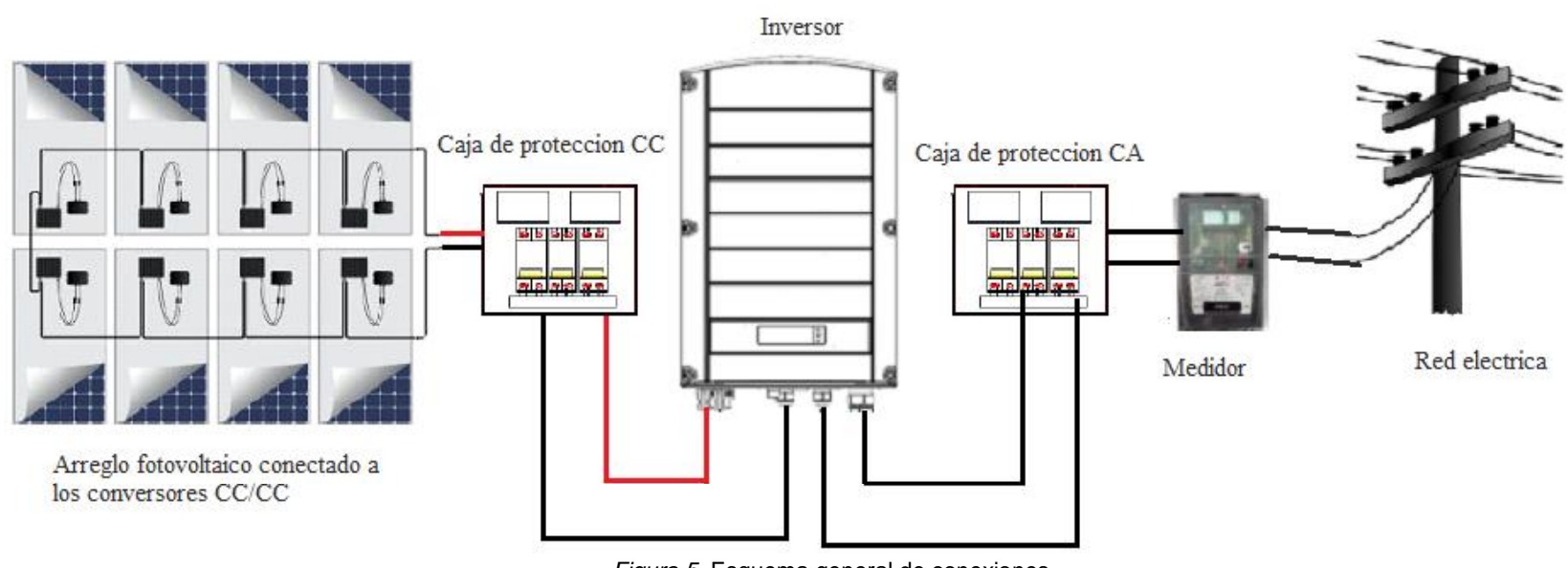

Figura 5. Esquema general de conexiones.

En la Figura se muestra el esquema general de conexionado de los módulos fotovoltaicos a los conversores cc/cc conectados en serie, luego a la caja de protecciones del lado $\mathrm{CC}$, seguidamente se conecta en paralelo con el inversor y a su respectiva caja de protección en el lado CA, y finalmente se conecta a la red. La conexión en serie permitirá la suma de voltajes, operando en condiciones estándares de prueba, serán $\mathrm{Pmp}=2.96 \mathrm{kWp}, \mathrm{Vmp}=$ $320.8 \mathrm{~V}, \operatorname{Imp}=9.23 \mathrm{~A}, \mathrm{Voc}=386.4 \mathrm{~V}$, Isc $=9.95 \mathrm{~A}$, conforme a los datos proporcionados por el fabricante.

EI SFCR necesitará de un conjunto de dispositivos de protección y de seccionamiento en el lado de corriente continua (CC) y de corriente alterna (CA), en conexión monofásica, los dispositivos de protección estarán compuestos de fusibles de 15A, interruptor termomagnético de 16A, 3 DPS de 1000V/40KA en el lado C.C., y 2 PDS de $275 \mathrm{~V} / 50 \mathrm{KA}$, disyuntor de $25 \mathrm{~A} \mathrm{e}$ interruptor termomagnético de $20 \mathrm{~A}$ en el lado C.A.

\section{Pérdidas por temperatura}

Para el cálculo del factor que considera las pérdidas medias mensuales debidas a la temperatura, "Ltem", se hace uso de la siguiente ecuación:

$$
\text { Ltem }=g \times\left(T c-25^{\circ} \mathrm{C}\right)
$$

Dónde:

g: Coeficiente de temperatura de Pmax. En nuestros módulos es $0.0057 \%\left(1 /{ }^{\circ} \mathrm{C}\right)$

Tc: Temperatura de los módulos.

Para encontrar la temperatura de los módulos se utiliza la siguiente ecuación:

$$
T_{c}=T a m b+\frac{\left(\text { Tonc }-20^{\circ} \mathrm{C}\right) x E}{800}
$$

Dónde:

Tamb: Temperatura ambiente

Tonc: Temperatura de operación nominal de la célula. En nuestro caso es de $45^{\circ} \mathrm{C}$.

E: Irradiación media en un día soleado, varía según el mes.

Sustituyendo los valores de la ecuación 2 en la ecuación 1 nos queda la siguiente ecuación:

$$
\text { Ltem }=0.0057 \times\left(\text { Tamb }+\frac{E}{32}-25^{\circ} \mathrm{C}\right)
$$

\section{Pérdidas en el conversor cc/cc y el inversor}

Las pérdidas de potencia en el inversor pueden ser interpretadas por la suma de tres componentes (Macedo, 2006):

Pérdidas de autoconsumo, $K_{0}=P_{\text {auto }}(\mathrm{W})$

Pérdidas lineales con la corriente (caída de tensión en semiconductores), $K_{1} P_{\text {salida }}$

Pérdidas proporcionales al cuadrado de la corriente (pérdidas óhmicas), $K_{2} P^{2}$ salida

$$
P_{\text {pérdidas }}=P_{\text {auto }}+K_{1} P_{\text {salida }}+K_{2} P_{\text {salida }}^{2}
$$

Dividiendo la ecuación (1) por la potencia nominal del inversor del inversor $P_{\text {inv }}$, resaltando que $p_{\text {salida }}=$ $P_{\text {salida }} / P_{\text {inv }}$ y considerando $k_{0}=P_{\text {auto }} / P_{\text {inv }}, k_{1}=K_{1} \mathrm{y}$ $k_{2}=K_{2} P_{\text {inv }}$, obteniéndose la siguiente ecuación (5).

$$
p_{\text {pérdidas }}=k_{0}+k_{2} p_{\text {salida }}+k_{2} p_{\text {salida }}^{2}
$$

La ventaja de la ecuación es que se puede caracterizar completamente el comportamiento de las pérdidas en el inversor y los conversores cc/cc, y consecuentemente su eficiencia en base a solo tres parámetros adimensionales $k_{0}, k_{1} \mathrm{y} k_{2}$. Para determinar los valores de los parámetros $k_{0}, k_{1}$ y $k_{2}$ se utilizan las siguientes ecuaciones (Martin, 1998).

Donde $\eta_{i n v 0,1}, \eta_{i n v 0,5}$ y $\eta_{i n v 1}$, y son valores de la eficiencia instantánea correspondientes a la cargas del inversor de $10 \%, 50 \%$ y $100 \%$ respectivamente, de la potencia nominal del inversor, estos valores pueden ser obtenidos de la curva de eficiencia del inversor.

$$
\begin{aligned}
& k_{0}=\frac{1}{9} \frac{1}{\eta_{i n v 1}}-\frac{1}{4} \frac{1}{\eta_{i n v 0,5}}+\frac{5}{36} \frac{1}{\eta_{i n v 0,1}} \\
& k_{0}=-\frac{4}{3} \frac{1}{\eta_{i n v 1}}+\frac{33}{12} \frac{1}{\eta_{i n v 0,5}}+\frac{5}{12} \frac{1}{\eta_{i n v 0,1}}-1 \\
& k_{2}=\frac{20}{9} \frac{1}{\eta_{i n v 1}}-\frac{5}{2} \frac{1}{\eta_{i n v 0,5}}+\frac{5}{18} \frac{1}{\eta_{i n v 0,1}}
\end{aligned}
$$

Para calcular la potencia de salida de los inversores, se utiliza el modelo de eficiencia de conversión del 
inversor el cual depende de la potencia de salida. Con base en los efectos físicos involucrados representados por $K_{0}, K_{1}$ y $K_{2}$ se propuso la siguiente ecuación (Macedo, 2006).

$n_{\text {ivv }}\left(p_{\text {salida }}\right)=\frac{P_{\text {salida }}}{P_{\text {entrada }}}=\frac{P_{\text {salida }}}{\left(P_{\text {salida }}+P_{\text {pertidas }}\right)}=\frac{P_{\text {salida }}}{p_{\text {salida }}+k_{0}+k_{1} p_{\text {salida }}+k_{2} p_{\text {salida }}^{2}}$

Tabla 4.

Coeficientes de pérdidas calculados y obtenidos experimentalmente (Teles, 2017).

\begin{tabular}{|c|c|c|c|c|c|c|}
\hline \multirow{2}{*}{$\begin{array}{l}\text { Sistemas / } \\
\text { Coeficientes }\end{array}$} & \multicolumn{3}{|c|}{ Calculado } & \multicolumn{3}{|c|}{ Experimental } \\
\hline & $K_{0}$ & $K_{1}$ & $K_{2}$ & $K_{0}$ & $K_{1}$ & $K_{2}$ \\
\hline Inversor & 9,403 & 0,016 & $2 \times 10^{-6}$ & 8,115 & 0,025 & $5 \times 10-7$ \\
\hline $\begin{array}{c}\text { Conversor } \\
\text { Cc/cc }\end{array}$ & 0,902 & 0,006 & $14 \times 10^{-6}$ & 0,178 & 0,021 & $7 \times 10-5$ \\
\hline $\begin{array}{c}\text { Conversor + } \\
\text { Inversor }\end{array}$ & & & & 18,7 & 0,0307 & $1 \times 10-5$ \\
\hline
\end{tabular}

Para calcular la eficiencia del inversor y de los conversores cc/cc se tomará en cuenta los datos obtenidos por Teles (2017), que trabaja con el inversor y los conversores cc/cc de la misma marca Solaredge.

\section{Cálculo de la producción anual esperada}

La producción de energía se calcula en función de la irradiación, la potencia instalada y el rendimiento energético de la instalación, según la siguiente ecuación:

$$
E=G * P \_n o m * P R
$$

Dónde:

$\mathrm{G}=$ Irradiación solar media

Pnom = Potencia Nominal

$\mathrm{PR}=$ Rendimiento energético

\section{Valor Presente Neto (VPN)}

$$
V P N=-I+\sum_{i=1}^{n} \frac{Q_{n}}{(1+r)^{n}}
$$

Donde $\boldsymbol{Q}$ n representa los flujos de caja, $\boldsymbol{I}$ es el valor del desembolso inicial de la inversión, $\boldsymbol{n}$ es el número de periodos considerados y $\boldsymbol{r}$ es la tasa de descuento a la cual se van a descontar los flujos efectivos (Beltrán et al., 2016).

\section{Tasa Interna de Retorno (TIR)}

$$
V P N=-I+\sum_{i=1}^{n} \frac{Q_{n}}{(1+r)^{n}}=0
$$

Donde $\boldsymbol{r}$ es igual a la TIR entonces se puede afirmar que la TIR representa la rentabilidad con respecto a una tasa mínima o de corte del inversionista (Beltran et al., 2016).

\section{Período de recuperación de la inversión}

$$
P R S=\mathrm{a}+\frac{I_{0}-b}{F_{t}}
$$

Donde a es el número del periodo inmediatamente anterior hasta recuperar el desembolso inicial $\boldsymbol{I}_{\mathbf{0}}$ es la inversión inicial del proyecto, b es la suma de los flujos hasta el final del periodo «a», Ft es el valor del flujo de caja del año en que se recupera la inversión.

\section{Relación Beneficio/ Costo}

$$
B / C=\frac{V A I}{V A C}
$$

Dónde: B/C es la relación costo-beneficio, VAI es el valor actual de los ingresos totales netos o beneficios netos y VAC es el valor actual de los costos de inversión o costos totales.

\section{Resultados y discusión}

\section{Análisis de los parámetros geográficos y climatológicos}

En este ítem se van a comparar datos obtenidos de la irradiación en el plano horizontal e irradiación en el plano inclinado, tenemos que elegir los datos con los que vamos a trabajar para calcular la producción anual esperada.

Tabla 5.

Irradiación en el plano en el plano inclinado. *(Condori Y. R. 2010), ** (Beltrán, N. et al 2019) Irradiación (kWh/m2/día)

\begin{tabular}{ll}
${ }^{\star}$ Según el modelo de Angstrom-Prescott & 6,5 \\
${ }^{* \star}$ Con celda solar calibrada & 6,0 \\
\hline
\end{tabular}

EI SFCR tendrá un $\beta=15^{\circ}$ y orientado al norte geográfico, según los datos proporcionados por la celda solar calibrada, se tiene un promedio anual de 6 $\mathrm{kWh} / \mathrm{m}^{2} / \mathrm{día}$

\section{Análisis por pérdida de temperatura}

Una vez ya conocidos los parámetros climatológicos y aplicando la ecuación (3), se obtiene los siguientes resultados: 
Tabla 6.

Pérdidas por temperatura

\begin{tabular}{|c|c|c|c|c|c|}
\hline Meses & $\operatorname{Tamb}\left({ }^{\circ} \mathrm{C}\right)$ & $\begin{array}{l}\text { GMedia } \\
(\mathrm{kWh} / \mathrm{m} 2-\mathrm{d})\end{array}$ & $\begin{array}{l}\text { Insolación real } \\
\text { (h) }\end{array}$ & $\begin{array}{c}E \\
(W / m 2-d)\end{array}$ & Ltem (\%) \\
\hline Enero & 17.85 & 5.62 & 12.37 & 454.32 & 4.0 \\
\hline Febrero & 16.70 & 5.61 & 12.12 & 462.87 & 3.5 \\
\hline Marzo & 18.45 & 5.45 & 11.46 & 475.57 & 4.7 \\
\hline Abril & 18.06 & 5.73 & 11.24 & 509.78 & 5.1 \\
\hline Mayo & 17.74 & 6.10 & 11.13 & 548.96 & 5.6 \\
\hline Junio & 17.37 & 5.81 & 11.17 & 519,25 & 4.9 \\
\hline Julio & 17.00 & 5.98 & 11.34 & 527,33 & 4.8 \\
\hline Agosto & 18.18 & 6.72 & 11.59 & 579.81 & 6.4 \\
\hline Setiembre & 19.34 & 6.29 & 12.25 & 513.46 & 5.9 \\
\hline Octubre & 19.02 & 6.96 & 12.48 & 557.69 & 6.5 \\
\hline Noviembre & 18.91 & 5.95 & 13.01 & 457.34 & 4.6 \\
\hline Diciembre & 19.42 & 5.64 & 12.56 & 449,04 & 4.8 \\
\hline
\end{tabular}

\section{Análisis por pérdida de potencia de salida en el conversor cc/cc y el inversor}

Reemplazando las pérdidas en la potencia de salida de acuerdo a la ecuación (9) se obtiene la eficiencia del inversor y de los conversores cc/cc, que es un valor muy cercano a la eficiencia que nos brinda la ficha técnica proporcionada por el fabricante.

\begin{tabular}{|c|c|c|}
\hline \multicolumn{3}{|c|}{ Calculado Experimental } \\
\hline Inversor & $97.5 \%$ & $97.1 \%$ \\
\hline Conversor cc/cc & $98.7 \%$ & $95.5 \%$ \\
\hline Conversor cc/cc + Inverso & - & $96.2 \%$ \\
\hline
\end{tabular}

\section{Cálculo de rendimiento energético o "performance ratio"}

El coeficiente de rendimiento energético de un SFCR, se define como la eficiencia de la instalación en condiciones reales de trabajo, que tiene en cuenta la dependencia de las pérdidas (ver tabla 8).

\section{Producción anual esperada}

Por la ecuación (10), considerando la potencia nominal de $2.96 \mathrm{~kW}$ que es la potencia real del generador fotovoltaico. La irradiación solar diaria y el rendimiento energético obtenemos la energía producida mensual y anual (ver tabla 10).

De la Tabla podemos concluir que en el mes de octubre obtendremos la mayor cantidad de energía de 550.47 kWh, y la menor producción de energía se da en el mes de febrero que es de $414.68 \mathrm{kWh}$.

\begin{tabular}{lcc}
$\begin{array}{l}\text { Tabla } 8 . \\
\text { Datos para el análisis económico }\end{array}$ & & \\
\hline \multicolumn{1}{c}{ Parámetro de entrada } & Unidad & Dimensión \\
\hline Generador fotovoltaico & $\mathrm{kWp}$ & 2.96 \\
Energía producida & $\mathrm{kWh} / \mathrm{año}$ & 5650.99 \\
Tarifa regulada & $\mathrm{S} / . / \mathrm{kWh}$ & 0.6545 \\
Inversión inicial & $\mathrm{S} /$. & 29417.55 \\
Tasa de descuento & $\%$ & 8.58 \\
Mantenimiento & $\%$ & 0.2 \\
Operación & $\%$ & 0.4 \\
Vida útil del SFCR & años & 25 \\
\hline
\end{tabular}

\section{Resultados de indicadores económicos}

La energía entregada a la red es de 5,650.99 kWh. La tasa de descuento anual de $8.58 \%$, se toma en cuenta un índice medio para sistemas fotovoltaicos. La vida útil del SFCR se toma en cuenta el máximo tiempo de uso de los módulos fotovoltaicos, conversores cc/cc y el inversor. El costo de la energía entregada por el SFCR es tomada a la misma tarifa regulada practicada por la empresa distribuidora de energía eléctrica, opción tarifaria BT5B Residencial, (ver tabla 11). 


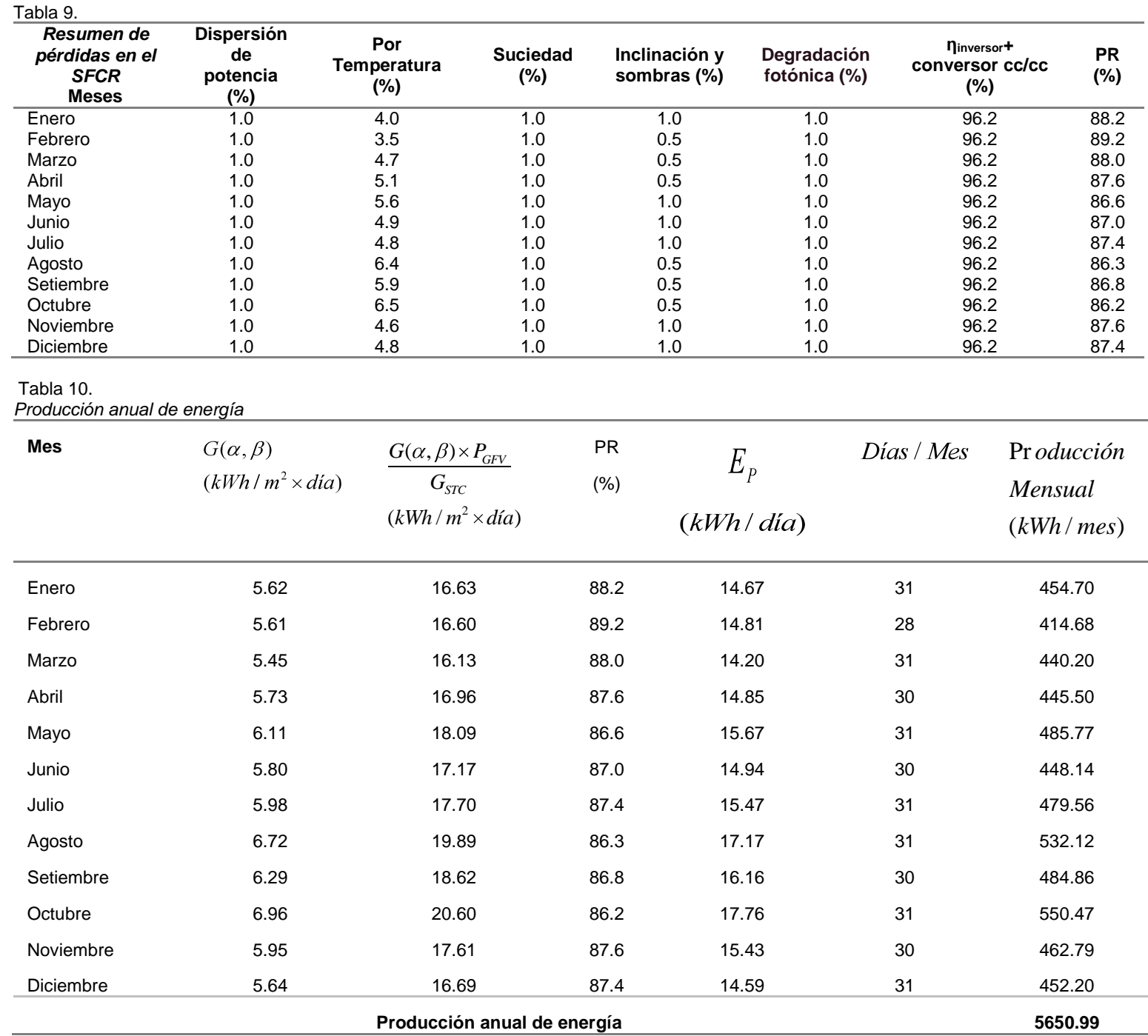

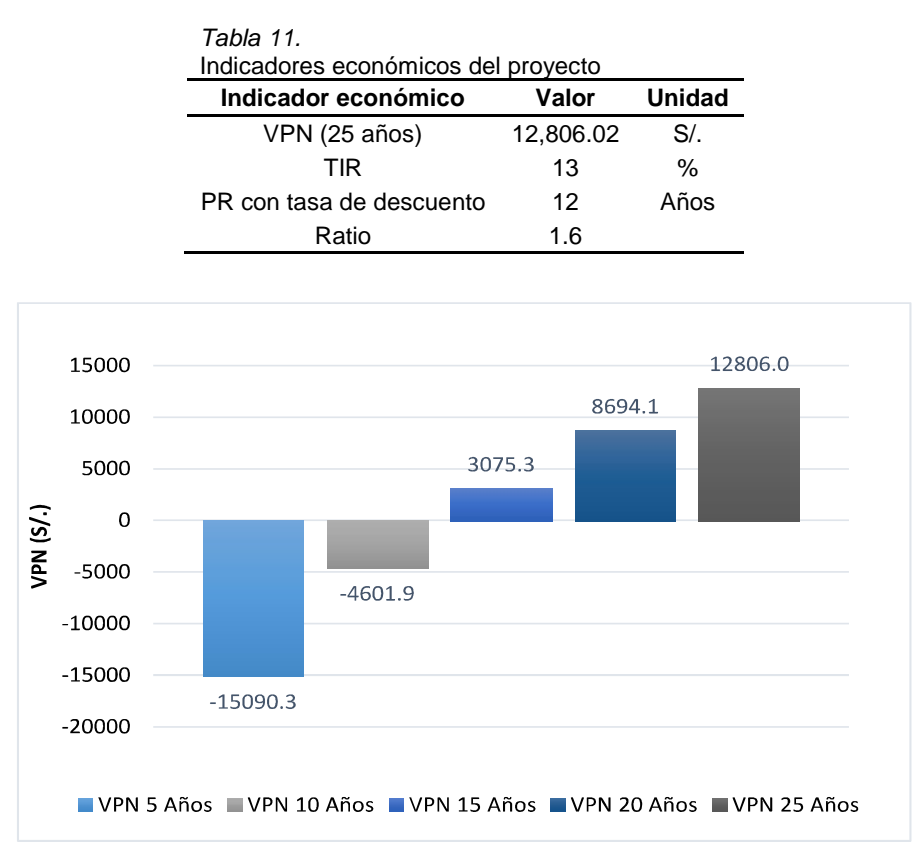

Figura 6. Valor Presente Neto (VPN) del SFCR de 3kW. 
Para el flujo de caja proyectado para 25 años, entre los egresos tenemos el pago del financiamiento y el costo de operación y mantenimiento, y entre los ingresos tenemos la venta de energía a la red. Aplicando las ecuaciones (11), (12), (13) y (14), recalcando que estos datos son calculados con tasa de descuento y considerando el valor del dinero en el tiempo, se tiene los siguientes resultados:

El periodo de recuperación con los periodos descontados al tiempo actual nos da como resultado 12 años, y el ratio es de 1.60, lo que significa que por cada 1.0 sol invertido, se tiene una ganancia de 0.60 centavos. Como el valor del VPN es positivo y el TIR $13 \%$, entonces se demuestra que el proyecto es viable financieramente.

\section{Implementación}

Estructura de soporte de fierro galvanizado, la cual deberá contar con las dimensiones y características definidas en la sección de dimensionamiento.

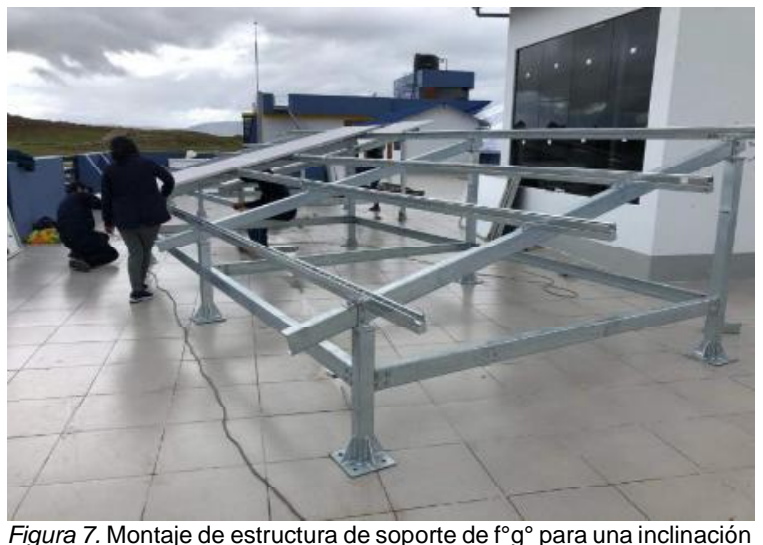
de $15^{\circ}$ y una orientación al NG

\section{Conclusiones}

Los conversores cc/cc corresponden a las nuevas alternativas distribuidas existentes en el mercado actual con un rendimiento experimental incluido el inversor de $96.2 \%$, con un PR de 87.36 promedio anual, para $6.0 \mathrm{HSP}$ promedio y $15^{\circ}$ de inclinación y orientado al NG, se espera obtener una producción de $5,650.99 \mathrm{kWh} / a n ̃ o$ y una transferencia de energía a la red de 3,618.57 S/. /año, con un Pay back de 8 años y un Pay back con descuento de 12 años.

Los componentes del SFCR son 8 módulos fotovoltaicos modelo BSP370M, 8 conversores cc/cc modelo P370 y un inversor monofásico modelo $\mathrm{SE} 3000 \mathrm{H}$ como elementos principales.

El lugar donde se implementó el SFCR con conversores cc/cc es el pabellón del Instituto de Energías Renovables y Eficiencia Energética en la Universidad Nacional de Juliaca (UNAJ) sede Ayabacas, estratégicamente se elige la terraza norte de la infraestructura, y es prácticamente libre de sombras durante todo el año.

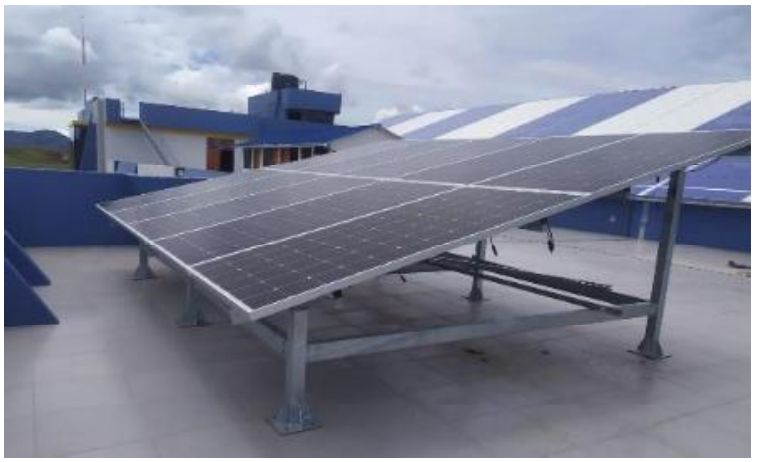

Figura 8. Montaje de paneles fotovoltaicos modelo BSP370M

Los resultados económicos muestran que el sistema es rentable recuperando la inversión inicial en el año 12 de funcionamiento; además se realizó el flujo de caja del proyecto para un tiempo de operación de 25 años, obteniendo para un precio de venta de energía de S/. 0.6545 KWh/mes, un VPN de S/. 12,806.02, un ratio de 1,60; y TIR de $13 \%$, podemos concluir que el proyecto es viable económicamente. 


\section{Referencias bibliográficas}

BAUER, E. (2019). Panel Solar 370W 72 Células Monocristalino.

Beltrán, N. B., Tinajeros, M., \& Morante, F. (2016). Analisis economico de un sistema fotovoltaico conectado a la red en Arequipa. 14-19.

Beltrán, N., Condori Y. R., Ramos, J. M., (2019). Proyecto de Investigación docente: "Diseño e implementación de un sistema SCADA para evaluación de parámetros de funcionamiento de un mini-parque solar fotovoltaico de $2.0 \mathrm{~kW}$ en sistemas aislados y conectados a la red para la UNAJ."

Corilla, D. R. (2014). Diseño de una planta solar fotovoltaicca de $200 \mathrm{KW}$ conectado a la red de media tension en el departamento de Ica.

Condori Yucra, R. (2010). Estudio para la climatización de la piscina y la produccion de agua caliente sanitaria acs con energia solar de la UNAP. Disponible en: https://docplayer.es/7039487-Estudio-para-laclimatizacion-de-la-piscina-y-la-produccion-deagua-caliente-sanitaria-acs-con-energia-solarde-la-unap.html

Creus Solé, A. (2014). Energias Renovables.

Domingo Cagigal, J. (2017). Simulacion en PSIM y SIMULINK de un conjunto rectificador con convertidor BUCK- BOOST en la salida.

ESAN. (2017, January 24). El índice beneficio/costo en las finanzas corporativas | Finanzas| Apuntes empresariales| ESAN. Retrieved June 19, 2020 from https://www.esan.edu.pe/apuntesempresariales/2017/01/el-indicebeneficiocosto-en-las-finanzas-corporativas/

Johns, M., Le, H., \& Seeman, M. (2008). GridConnected Solar Electronics. 1-12.

Lopes, L. C. (2006). Sistema experimental fotovoltaico de geracao de energia eletrica operando em paralelo com a rede eletrica CA. https://doi.org/10.1017/CBO9781107415324.00 4

Macedo, W. (2006). Análise do Fator de Dimensionamento do Inversor Aplicado a Sistemas Fotovoltaicos Comectados à Rede.

OSINERGMIN, O. S. de la I. en E. y M. (2017). La industria de la energia renovable en el Peru: 10 años de contribuciones a la mitigacion del cambio climatico.

Perpiñán, Ó., Colmenar, A., \& Alonso, M. (2012). Diseño de Sistemas Fotovoltaicos.

Solar Edge. (2019). Optimizador de Energía de SolarE dge.

Solaredge. (2019). 1.5-6kw Single phase inverter. Retrieved from https://www.thinkpower.com.cn/products_detail2-3.html

Teles, M. (2017). Avaliação operacional das diferentes arquiteturas de interligação de geradores fotovoltaicos à rede elétrica. 


\section{Agradecimientos}

A CONCYTEC-FONDECYT por el soporte financiero en convenio con el Banco Mundial, mediante el contrato $\mathrm{N}^{\circ}$ 180-2018-FONDECYT-BM-IADT-AV, para realizar este trabajo. Además, a la Universidade Federal de Pará (Grupo GEDAE) Belém-Brasil, Universidad Nacional de Juliaca (Grupo GRIDSOLAR) por el esfuerzo y dedicación que hicieron que este proyecto sea una realidad. 